\title{
Enterococcus dispar
}

National Cancer Institute

\section{Source}

National Cancer Institute. Enterococcus dispar. NCI Thesaurus. Code C86366.

A species of facultatively anaerobic, Gram-positive, cocci shaped bacteria in the phylum

Firmicutes. This species is nonmotile, non-spore forming, catalase negative, and

hydrolyzes esculin. E. dispar has been isolated from human specimens. 